\title{
Multistep direct preequilibrium reactions and the Monte Carlo approach
}

\author{
M.B. Chadwick ${ }^{1}$, M. Blann ${ }^{2}$, and S. Hilaire ${ }^{3}$ \\ 1 Group X-1, Applied Science and Methods, Los Alamos National Laboratory, NM 87545, USA \\ 2 San Diego, CA, USA \\ 3 CEA, Bruyères-le-Châtel, France
}

\begin{abstract}
We provide an overview of recent developments to the Hybrid Monte Carlo Simulation (HMS) model for calculating preequilibrium reactions. Illustrative examples are shown for nucleon and cluster induced reactions, and for assessing the importance of multiple preequilibrium processes. We present new results using HMS theory to study heavy nuclide recoil velocities, which are important in applications such as radiation heating, damage and single-event-upsets, and also discuss GNASH and TALYS code recoil predictions. We also discuss approaches for performing center-of-mass to laboratory kinematic transformations for the light-ejectile particles - we emphasize that correctly following the kinematics of sequentially decaying nuclei leads to results that can be quite different from those obtained using the traditional 2-body assumption for the recoil boost velocity. We also discuss how angular momentum transfer effects can be included semiclassically in the HMS model, allowing HMS to be linked to a Hauser-Feshbach code to study spin-dependent observables (such as gamma-ray, and isomer production).
\end{abstract}

\section{Introduction}

In recent years a Monte Carlo preequilibrium model has been developed that provides certain advantages for use in modeling nuclear reactions and generating evaluated ENDF databases. The initial formulation by Blann [1] was subsequently expanded to include a treatment of ejectile angular and energy distributions [2], and a treatment of cluster-induced reactions [3]. This approach is valuable because of its ability to accurately model a comprehensive variety of nuclear reaction mechanisms that occur for projectiles with incident energies up to a few hundred $\mathrm{MeV}$, as discussed below. Additionally, we focus below on the advantages of the Monte Carlo approach for modeling the velocities of heavy recoil nuclei produced in reactions - important for calculating radiation damage, heating, singe-event upsets, etc., as well as for correctly calculating $\mathrm{cm}$ to lab kinematic transformations for the light ejectile particles. We also describe our semiclassical approach for modeling spin transfer in the preequilibrium phase of a nuclear reaction, which is important when coupling the preequilibrium model to Hauser-Feshbach compound decay codes. Presently two implementations of this approach exist: Blann's Monte Carlo version of ALICE; and Chadwick's DDHMS code that has been released to collaborators participating in the NEA's Working Party on Evaluation Cooperation (WPEC) Nuclear Models working group.

Precompound models based on an assumed equilibration sequence of intranuclear two body interactions have proven valuable as a tool in the interpretation of a variety of nuclear reactions [4-6]. These models have in common a summation of contributions from ever more complicated (in terms of particle-hole excitations) configurations contributing to the decay process of the excited nuclei, prior to achieving a quasiequilibrium.

It has been demonstrated that for nucleon induced reactions, the three quasi-particle excitation is produced with nearly equal a-priori energy sharing between the three excitons, if calculated using N-N scattering cross sections in a Fermi gas [7]. Thus for the first term in the usual precompound decay models, the use of partial state densities based on exciton number [8] is justified. However, Bisplinghoff [9] clearly illustrated that precompound models in use were inconsistent in their use of higher order (than the first 3 exciton result) exciton density prescriptions, if the two body transition assumption was made. A second shortcoming of existing precompound models was a difficulty in treating multiple emission of precompound nucleons from a single nucleus, and in treating exclusive reactions for differential cross sections.

Both the aforementioned shortcomings of preequilibrium models have been removed, for nucleon (and heavy ion) induced reactions by application of Monte Carlo algorithms, in what we have called the Hybrid Monte Carlo Simulation (HMS), or more accurately the Precompound Monte Carlo Simulation model [1-3]. The latter name is preferable as the treatment is not a Monte Carlo version of the Hybrid model [10]. Rather it uses the fact that the 3 exciton configuration produced by the interaction of a nucleon with a nucleus in a two body process should give approximately the nucleon energy distribution represented by the 3 exciton density function [8], and by difference, of the two exciton density. In the Monte Carlo approach [1], each successive scattering of a nucleon is treated as producing a new 3 -exciton configuration, consistent with the 2-body assumption. This avoids use of the higher order exciton densities which Bisplinghoff demonstrated were inconsistent with population by a two body mechanism. This Monte Carlo approach may be used to calculate unlimited multiplicities of precompound emitted nucleons and may have various "gates" put on emitted nucleons. In other words, our Monte Carlo approach allows unlimited emission of preequilibrium ejectiles (so-called multiple preequilibrium), and it can be used to compute exclusive reactions where specific correlations between the ejectiles are of interest. 

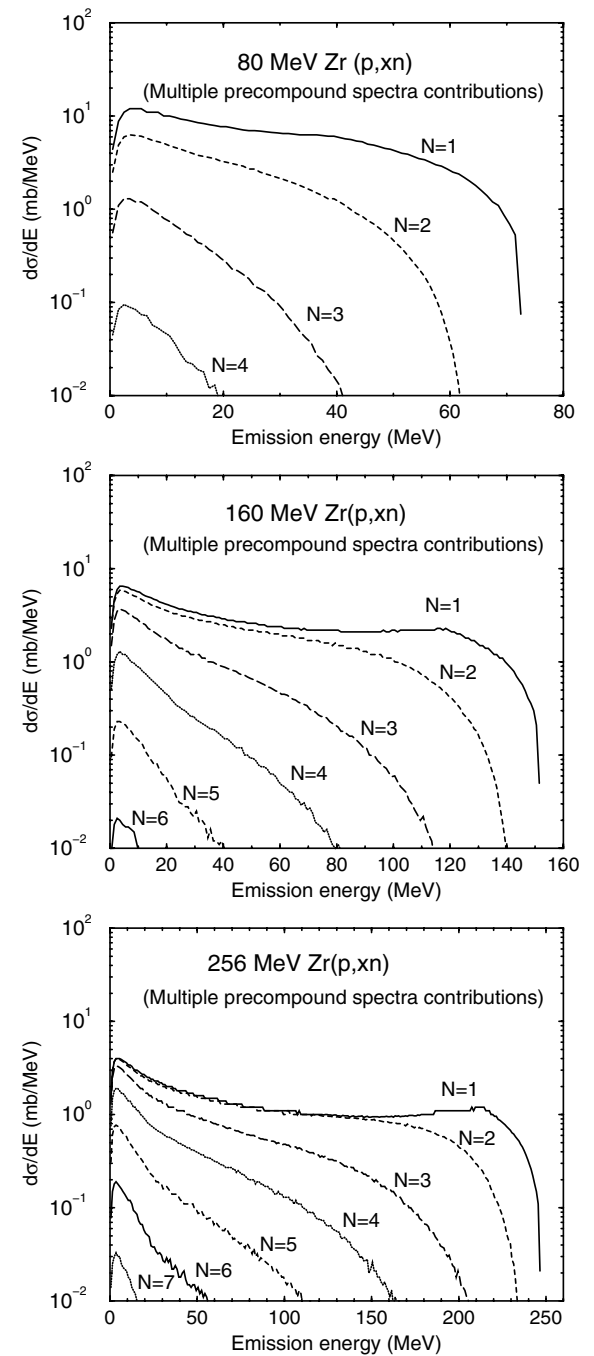

Fig. 1. Calculated preequilibrium spectra, including contributions from multiple preequilibrium processes.

\section{Background}

Before discussing our new results on recoil velocities, it is useful to review some of the results that have been obtained on preequilibrium emission spectra using the HMS model. The HMS model can be used to calculate preequilibrium reactions for incident energies of a few $\mathrm{MeV}$ up to a few hundred $\mathrm{MeV}$.

An example of angle-integrated spectra for 80, 160, and $256 \mathrm{MeV}$ protons incident on zirconium is shown in figure 1 , illustrating the increasing importance of multiple preequilibrium emission with incident energy. The figure shows spectra emitted following zero to $N-1$ other precompound nucleons. The increasing importance of precompound nucleons after the second (i.e., those emitted after two other nucleons) is clear. At $80 \mathrm{MeV}$, emission beyond two precompound nucleons is below the $10 \%$ level (which could be important for activation yields, but not too worrisome for single differential spectra). At $160 \mathrm{MeV}$, precompound spectra beyond multiplicity two are at the $30 \%$ or higher level at the lower range of the precompound spectra. As the incident proton energy increases, the relative contributions from multiplicity greater than two continues to increase, as is shown in the $256 \mathrm{MeV}$ results. We

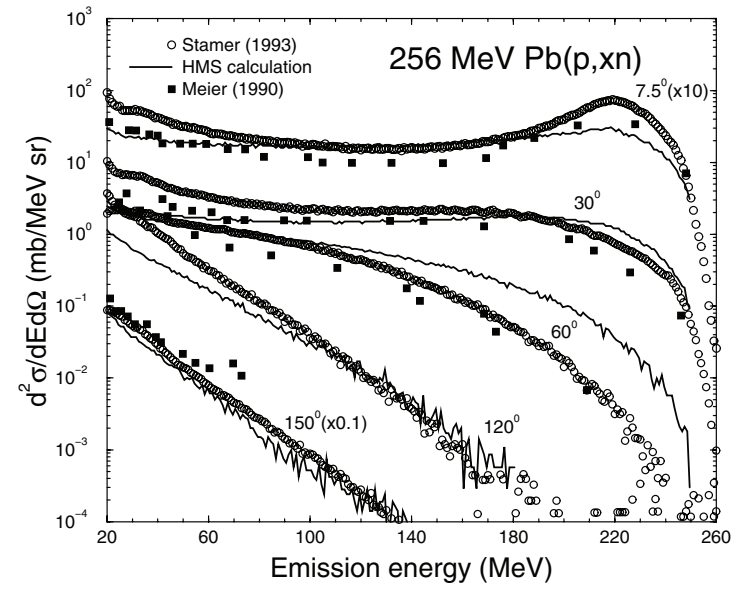

Fig. 2. Neutron emission spectra at various angles for $256 \mathrm{MeV} \mathrm{p}+$ ${ }^{208} \mathrm{~Pb}$, compared with data.

would emphasize that the " $N=$ " numbers in figure 1 do not represent exciton numbers in terms of older exciton models; all emissions in the HMS model are treated with respect to $2 p 1 h$, or $1 p 1 h$ configurations. The " $N=$ " numbers represent only the fact that there have been $N-1$ precompound nucleons emitted prior to the one for which the spectrum is being shown. The $N=1$ and $N=2$ results have a very high probability of representing the two nucleons from the initial scattering event, but either or both could also result from nucleons which have rescattered one or more times - and this will influence the angular distributions calculated. As the incident energy increases, we expect the $N=1$ and $N=2$ results to approach each other, except that the $N=2$ result must have its maximum energy reduced from that of $N=1$ by the binding energy of the first nucleon. This represents the fact that these emissions come primarily from the first scattering event, for which the energy partition is symmetric between the two particles after scattering, with the binding energy difference showing on the nucleon designated as the second to be emitted.

An example of HMS predictions of emission spectra at various angles, compared with measurements, is shown in figure 2. These data show secondary neutron spectra when $256 \mathrm{MeV}$ protons are incident upon ${ }^{208} \mathrm{~Pb}$. The general features of the experimental spectra appear to be reproduced by theory (though there is an over-prediction of the data at 60 degrees). The theory not only accounts for the energy-dependence of the spectra, but also the forward-peaking of the preequilibrium ejectiles which increases with increasing emission energy.

To illustrate the use of the HMS theory for modeling cluster induced reactions, we show in figure 3 results for incident alpha energies of 30.5 [11], 42 [12], and $55 \mathrm{MeV}$ [13], on niobium. For calculated results we have used the total reaction cross section as a starting point, without reduction. But for alpha induced reactions, it has been demonstrated that there are significant low momentum transfer reactions of type $\left(\alpha, \alpha^{\prime} \mathrm{x}\right)$, presumably due to the higher partial waves of the entrance channel. In a detailed analysis of these excitation functions, Gadioli et al. [14] conclude that only $60-70 \%$ of the reaction cross sections lead to the collisions in which the alpha might "dissolve" in the field of the nucleus. Also, it 


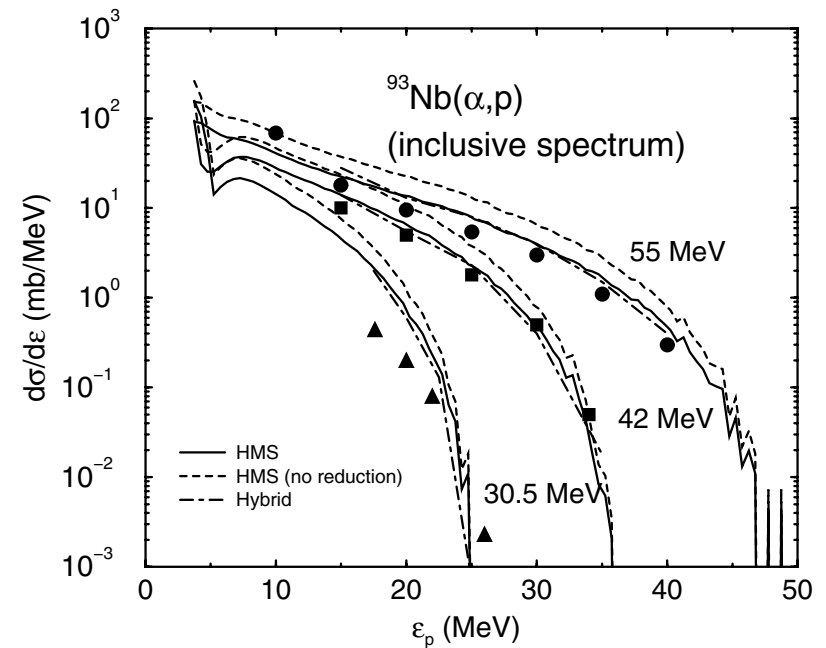

Fig. 3. Experimental and calculated $(\alpha, \mathrm{p})$ spectra on ${ }^{93} \mathrm{Nb}$. Triangles are points for $30.5 \mathrm{MeV}$ incident alpha particles from [11]; squares are for $42 \mathrm{MeV}$ alpha particles from [12], and circles are for $55 \mathrm{MeV}$ alpha particles from [13]. Solid lines are the results of the HMS Monte Carlo simulation described in this work, having been reduced by a factor 0.6 to account for reaction flux estimated to be lost to other low-momentum transfer reactions, (see text, and discussions by Gadioli et al. [14]). The HMS results without any such reduction are shown as dashed lines. Additionally, the dashed-dot lines show results using the hybrid model presented in ref. [5].

has been found empirically [5] that for odd A targets, alpha induced reactions are best represented by initial 5 exciton configurations. Results presented therefore were calculated assuming a 3 proton, 2 neutron set of initial excitons, i.e., it is assumed that the unpaired proton in the target was excited in the initial interaction. This is pure conjecture motivated by the empirical observations of past analyses of alpha induced reactions.

We see excellent agreement between calculated and experimental results for the shapes of the spectra at 42 and $55 \mathrm{MeV}$ incident alpha energy; if the reaction cross sections were reduced by $40 \%$ consistent with the conclusion of Gadioli et al., the magnitudes would be in excellent agreement with the experimental results. The agreement with the $30.5 \mathrm{MeV}$ incident alpha data is poor both respect to shape and magnitude; however in terms of the experimental uncertainties of the 4 measured points, the calculated results are not unreasonable.

\section{Accurate $\mathrm{cm} \rightarrow$ lab transformations and recoils}

For reactions at energies where more than one particle may be emitted, most statistical nuclear model codes use an approximate method for performing center-of-mass to laboratory kinematical transformations, whereas the Monte Carlo method allows these transformations to be performed exactly. Previous approaches typically take a model calculation performed in the channel energy frame (the combined kinetic energy of the light ejectile and the recoil), and boost to the lab frame with a velocity boost derived from the projectile (beam) momentum and composite nucleus mass. Whilst this is accurate for the first emitted ejectile, it is not correct for

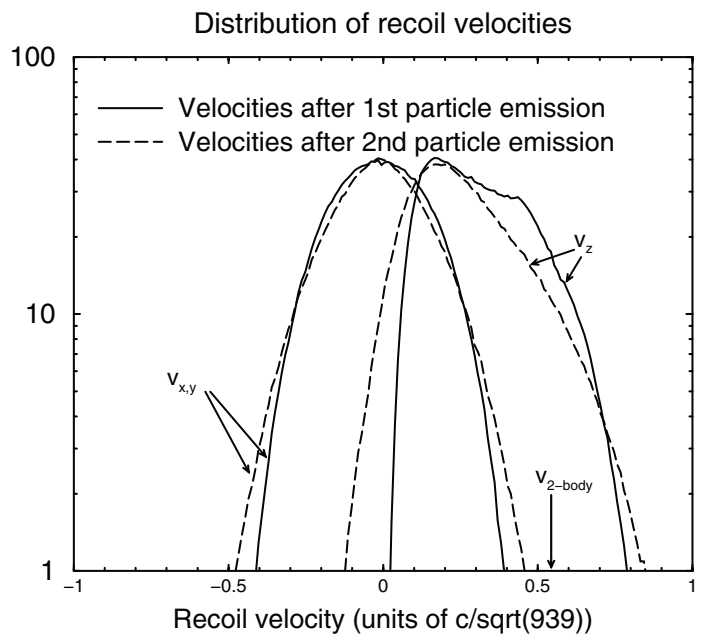

Fig. 4. Recoil velocities in the $\mathrm{x}, \mathrm{y}, \mathrm{z}$ directions after $113 \mathrm{MeV}$ protons in the z-direction impinge upon ${ }^{27} \mathrm{Al}$, after one and two light particles (preequilibrium or equilibrium) are emitted. The vertical arrow labeled $v_{2 \text {-body }}$ shows the traditional recoil velocity often erroneously assumed for $\mathrm{cm} \rightarrow$ lab transformations based on a 2body assumption for the moving composite system.

those subsequently emitted . Each of these ejectiles is emitted from a moving (excited) nucleus, and the kinematical boost should account for the velocity $(\mathrm{Vx}, \mathrm{Vy}, \mathrm{Vz})$ of that moving nucleus - and not the traditionally used value based on target mass and beam momentum. A Monte Carlo preequilibrium and equilibrium decay model allows the velocity components of the excited decaying nuclei to be followed at each stage of the emission process so that correct kinematical boosts can be performed.

An example is shown in figure 4 for the recoil velocity distributions of heavy nuclei after the emission 1 and after 2 light particles, in $113 \mathrm{MeV} \mathrm{p}+{ }^{27} \mathrm{Al}$ reactions. These distributions were obtained by gating on any particle type (neutrons or protons), and any emission mechanism type (preequilibrium or equilibrium), though preequilibrium emission dominates for the first particle out. The figure also shows the moving composite system velocity traditionally used for $\mathrm{cm} \rightarrow$ lab kinematic boosts (labeled " $v_{2 \text {-body }}$ "). It is evident that the peak of the recoil velocity distribution in the z-direction after 1 and 2 particles have been emitted lies at a significantly lower velocity than the traditional value. Also, whilst velocities in the $\mathrm{x}$ and $\mathrm{y}$ direction are traditionally taken as zero, we see a rather broad range of values (but with a mean of zero).

This effect is particularly important as the incident energy increases, since the preequilibrium particles are typically emitted with high-energy in the forward direction. In this case the excited residual nucleus after preequilibrium emission moves with a much slower recoil velocity than that of the traditional approximation. Using recoil velocities based on the kinematic history of the event to boost subsequent decay particles is important for obtaining correct laboratory frame angular distributions for the low-energy ejectiles. The effect is most pronounced for light target nuclei, where using just one initial composite system velocity boost for all ejectiles can significantly distort the calculated laboratory angular distributions. 


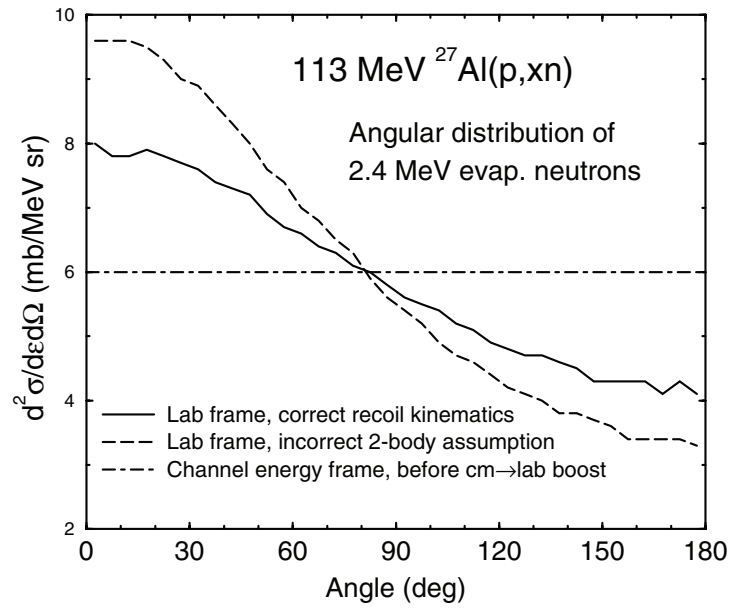

Fig. 5. Angular distribution of $2.4 \mathrm{MeV}$ evaporation neutrons emitted after $113 \mathrm{MeV}$ protons impinge upon ${ }^{27} \mathrm{Al}$. Distributions are shown for the laboratory frame, and also for the channel frame before the $\mathrm{cm} \rightarrow$ lab boost.

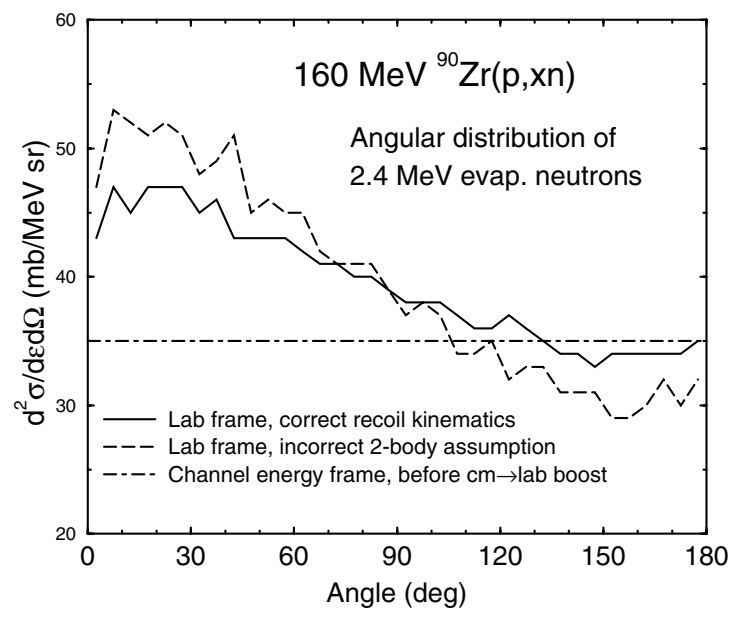

Fig. 6. As for figure 5 but for $160 \mathrm{MeV} \mathrm{p}+{ }^{90} \mathrm{Zr}$.

This can be seen in figures 5 and 6 where we show the angular distribution of emitted evaporation neutrons, at $2.4 \mathrm{MeV}$, for 113 protons incident on ${ }^{27} \mathrm{Al}$, and $160 \mathrm{MeV}$ protons incident on ${ }^{90} \mathrm{Zr}$. We show 2 figures for a light and for a heavier target nucleus to illustrate that the effect is present not only for light targets. The figures show the calculated angular distributions using both the correct kinematic history of the event with correct recoil velocities, and using the traditional 2-body recoil assumption. The "correct" angular distribution is seen to be significantly less forward-peaked in the laboratory frame due to the slower recoil velocities after preequilibrium. The figures also show the assumed isotropic angular distributions in the channel energy frame before the $\mathrm{cm} \rightarrow$ lab transformations. Interestingly even for a mediumheavy target nucleus $\left({ }^{90} \mathrm{Zr}\right.$, fig. 6) we see a significant effect (the fluctuations in the results shown are statistics from the Monte Carlo calculation).

This conclusion influences how we should represent these reactions in ENDF-6 format. At present most groups like to store angle-integrated dxs emission spectra (energy-dependent) in the $\mathrm{cm}$ frame, together with Kalbach preequilibrium ratios, from which a transport code subsequently performs $\mathrm{cm} \rightarrow$ lab transformations (with a velocity boost based on the projectile momentum). This approach enables the production of very compact ENDF files since full ddxs spectra are not tabulated. However, our above discussion shows that this approach introduces biases into the labframe results that may be unacceptably high; if so then the ENDF file may instead have to tabulate full ddxs lab-frame spectra. Alternatively, it may be possible to develop other approaches that preserve the basic advantages of tabulating energy-dependent dxs spectra with Kalbach preequilibrium ratios, but that address (perhaps in an approximate way) the need to use lower recoil boost velocities after preequilibrium emission.

At present our HMS preeuilibrium module is not integrated fully into the GNASH code [15], and so full estimates of the recoil velocity and kinetic energy distributions await the coupling of the HMS and Hauser-Feshbach GNASH modules. Instead, at present we calculate recoils using a different approach within GNASH, developed by Chadwick in the late 1990s. This approach tracks the kinetic energy transfers to the heavy excited nuclear system as (multi) particle emission occurs, until the ground state final residual nucleus is created. Certain approximations were made for modeling situations where many decay pathways can contribute to the same final decaying residual nuclues. These are described in detail in ref. [16].

This approach can be contrasted to that recently incorporated into the NRG-Bruyères-le-Châtel TALYS code by Hilaire and Koning [17]. Two hypothesis have been considered in TALYS, and the results for $100 \mathrm{MeV} \mathrm{n}+{ }^{28} \mathrm{Si}$ reactions are shown in figure 7 for two representative recoil nuclei, compared with GNASH predictions. In the case labelled as TALYS 1.0 (Best), the entire velocity distribution of the compound nuclei is taken into account while calculating the various light particle emissions and consequently the associated recoils. In the case labelled as (Simple), the average value of the velocity distribution is used (an assumption also made in the simpler GNASH calculations here). This means that each time a compound nucleus is decaying, we assume that this nucleus has a definite $\mathrm{CM}$ velocity. Of course, because of the extra loops that are necessary in the (Best) case, the calculation time is more important. In the attached figures, in the Best situation the calculation took 25 minutes and 6 minutes for the Simple case. Both calculations have been performed with 10 bins to store the recoil spectra. We can of course increase this number of bins, but the computation time will increase correspondingly. The different approaches are seen to be qualitatively similar, but differ in the details predicted. The differences reflect not only the different algorithms developed to predict recoil velocities, but also overall differences in the TALYS and GNASH predictions for partial cross section channels in this reaction.

\section{Preequilibrium spin transfer}

In many applications it is important to know the angular momentum distribution of excited nuclei after the preequilibrium phase of the reaction. When coupling a preequilibrium 

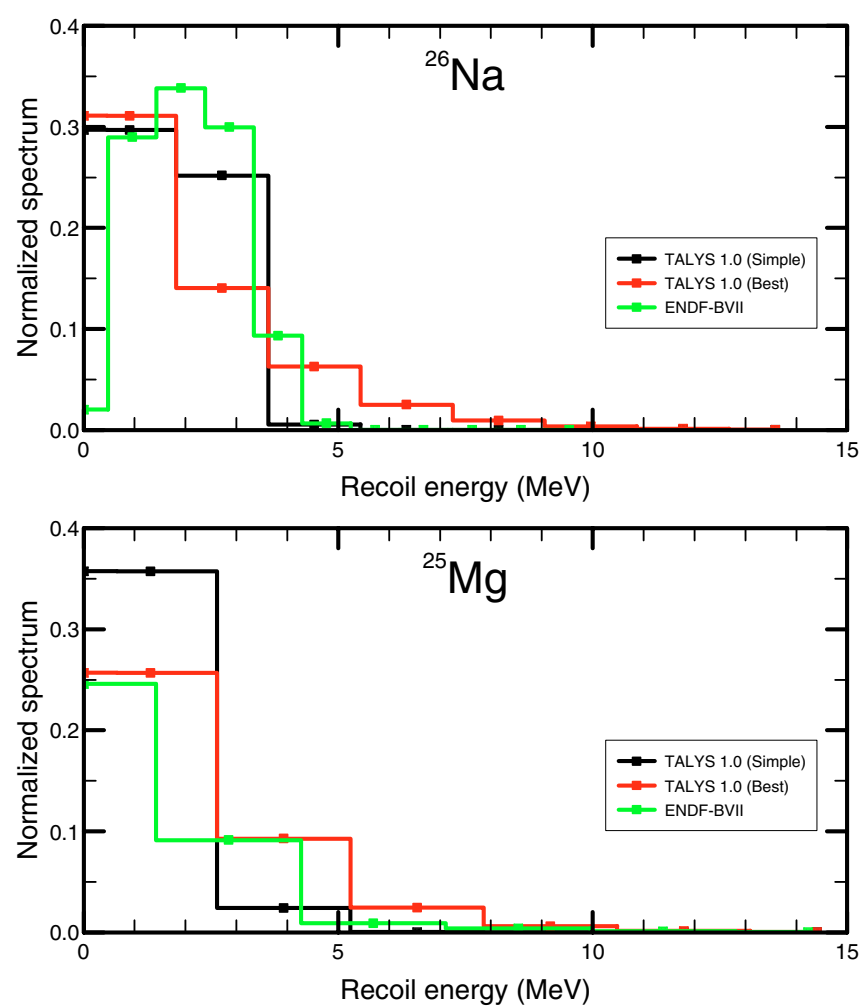

Fig. 7. Comparison of TALYS and GNASH recoil spectra (labeled ENDF) predicted for two representative recoil nuclides following $100 \mathrm{MeV} \mathrm{n}+{ }^{28} \mathrm{Si}$ reactions.

model to a Hauser-Feshbach code that handles the decay of excited compound nuclei (and conserves both spin and parity), it is necessary to know the initial spin values of the decaying nuclei. This is important in applications which are particularly sensitive to angular momentum phsyics, such as modeling the production of spin isomers in residual nuclei for activation studies, and modeling discrete gamma-ray line cross sections in the decay of residual nuclei for elucidating nuclear reaction mechanisms (e.g. the focus of the GEANIE experimental program at Los Alamos' LANSCE/WNR facility). It can also be important in reactions that lead to fission since the fission barrier heights depend on the compound nucleus' angular momentum.

In a semiclassical context, the HMS Monte Carlo preequilibrium model can be used to estimate the spin transfer to the residual nucleus in preequilibrium reactions. This has been done in both Blann's ALICE Monte Carlo code, and in Chadwick's DDHMS code (in collaboration with Pavel Oblozinsky). The approach estimates the angular momentum transfer in terms of rxp. To determine the impact parameter, two options are available in DDHMS: (1) sample from a distribution based on a Fermi distribution, as for instance defined in Blann's Geometry-Dependent Hybrid model; (2) sample from a distribution that, in a semiclassical sense, reproduces (or "mimics") the $l$-distribution from an optical potential that is used as an input into the calculations. The latter approach has the advantage that it guarantees agreement with a HauserFeshbach compound nucleus spin distribution in the limiting case of no preequilibrium emission. The algorithms used also

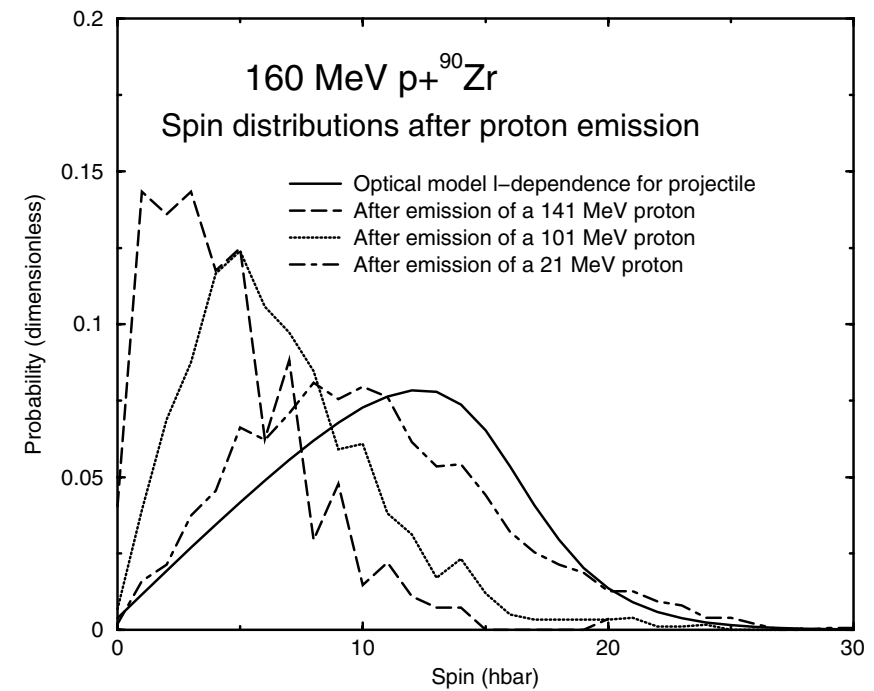

Fig. 8. Spin distributions of residual nuclei after a preequilibrium proton is emitted in $160 \mathrm{MeV} \mathrm{p}+{ }^{90} \mathrm{Zr}$ reactions, obtained from the DDHMS code. The initial orbital angular momentum distribution brought in by the projectile is also shown as a solid line. Fluctuations in the calculated results are due to Monte Carlo statistics.

account for the orbital angular coupling to intrinsic target, projectile, and ejectile spins.

Figure 8 shows residual nucleus spin distributions after a preequilibrium proton is emitted in $160 \mathrm{MeV} \mathrm{p}+{ }^{90} \mathrm{Zr}$ reactions, obtained from the DDHMS code version 1.10. The initial orbital angular momentum distribution brought in by the projectile is also shown as a solid line. As the emission energy decreases the angular momentum carried away by the ejectile decreases, leaving a residual nucleus spin distribution that tends to values close to the initial orbital angular momentum brought in by the projectile. As the emission energy increases, corresponding to typically forward-peaked angular distribution preequilibrium particles, the spin distribution of the residual nucleus is peaked at increasingly low spins, as would be expected. We have compared these results against those obtained from Mashnik's CEM2k intranuclear cascade code, and see qualitative agreement.

To illustrate spin distributions following preequilibrium reactions at lower incident energies, figure 9 shows spin distributions after a preequilibrium neutron is emitted in $14 \mathrm{MeV}$ $\mathrm{n}+{ }^{179} \mathrm{Hf}$ reactions, also obtained from the DDHMS code version 1.10. The initial orbital angular momentum distribution brought in by the projectile is also shown as a thin solid line. Here the target spin is so large (4.5) that the compound nucleus spin for ${ }^{180} \mathrm{Hf}$ (bold solid line) is boosted to higher spins compared to the orbital distribution. The result from the DDHMS calculation (when no preequilibrium emission occurs) agrees well with the result obtained from HauserFeshbach theory (dot dash) using the GNASH code. The spin distribution following preequilibrium emission of a $8.5 \mathrm{MeV}$ neutron is shown as a long-dashed line. In general, we expect preequilibrium to lead to spin distribution peaked at lower values. This is not clearly seen here because the energies are too low, and because of the effects of coupling to the high target spin, but there is some indication of a shift down in spin for the peak of the distribution. The dotted line shows the spin 


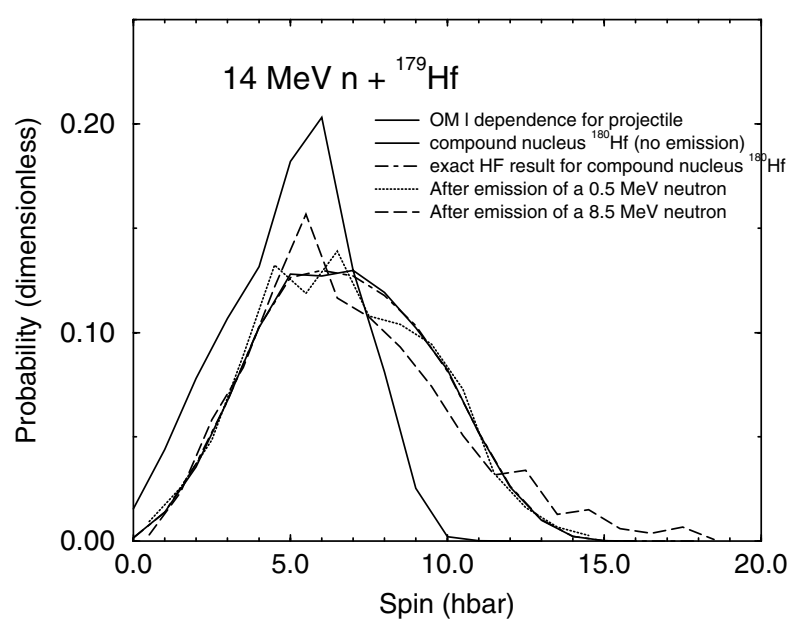

Fig. 9. Spin distributions of residual nuclei after a preequilibrium neutron is emitted in $14 \mathrm{MeV} \mathrm{n}+{ }^{179} \mathrm{Hf}$ reactions, obtained from the DDHMS code. The initial orbital angular momentum distribution brought in by the projectile is also shown as a thin solid line. Fluctuations in the calculated results are due to Monte Carlo statistics.

distribution after emission of a low energy $(0.5 \mathrm{MeV})$ neutron - as expected, this is hardly changed from the initial (bold solid line) spin distribution, since the ejectile carries out very little angular momentum.

\section{Isomer ratio calculations}

We are undertaking some research work for the Los Alamos Accelerator Transmutation of Waste (ATW) program, where we are producing neutron and proton activation nuclear data libraries up to $150 \mathrm{MeV}$. These libraries contain cross sections for the production of all nuclear productions, as excitation functions, for over 650 stable target nuclei, and can be used in a burnup code such as CINDER to calculate the timedependent transmutation and radioactivity in an ATW system. An initial version of this activation file was produced a few years ago by Koning and Chadwick, based on HMSALICE calculations of isotope production, and in the case of incident neutrons the results were used to augment the European Activation File (1997) evaluated data below $20 \mathrm{MeV}$.

However, the HMSALICE calculations used in this earlier activation file were not able to predict information on isomer production, since angular momentum considerations were not included in the original code. Below we describe our recent developments to model angular momentum transfer, and hence isomer production. The work described is work-in-progress. Because such large scale calculations have to be performed for hundreds of target nuclei, we have focused on methods that are computationally fast to implement.

Of the several options just referred to, we are beginning isomer yield calculations in HMSALICE using the entrance channel spin distribution from the optical model as starting point. Thus if the entrance channel has partial waves "I" with partial cross sections $\sigma(\mathrm{I})$, we may divide the total number of cascade events into a number $\mathrm{N}(\mathrm{I})=N_{\text {cas }} \cdot \sigma(\mathrm{I}) / \sigma_{R}$, where $/ \sigma_{R}$ is the total reaction cross section from the optical model and $N_{c a s}$ is the total number of cascades to be executed.

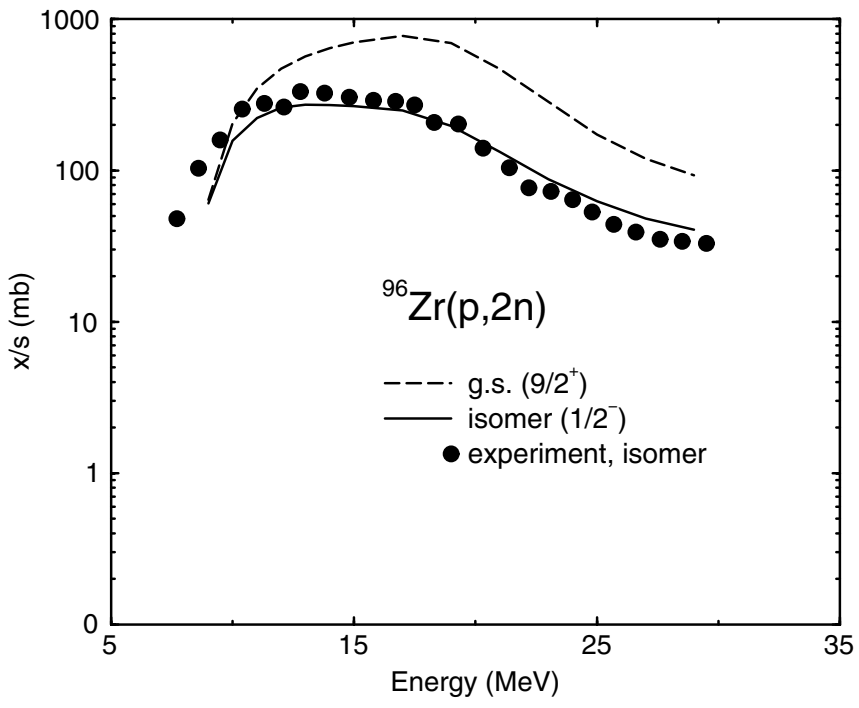

Fig. 10. Calculated production of the isomer and ground state.

For entrance channel with spin "I", when we have calculated the emission energy $e$ and angle $\theta$ for preequilibrium or equilibrium emission, we can calculate a decrement to the initial angular momentum "I" as

$$
\Delta I=I \cdot \cos (\theta) * \sqrt{\left(2 . * e . \mu_{\text {eject }}\right)} / \sqrt{\left(2 . * e_{\text {incident }} \cdot \mu_{\text {beam }}\right)}
$$

where $e_{\text {incident }}$ is the beam energy, $\mu_{\text {eject }}$ is the ejectile mass, and $\mu_{\text {beam }}$ is the beam mass. This is basically an argument that the spin removed is given by rxp, where "p" is the ejectile momentum, and we assume that the radius of emission $r$ is the same as given by the entrance channel "I". We sum the $\delta$ I over all ejectiles in each cascade, until an excitation is reached for which no further particle emission is energetically possible. The final spin after particle emissions is set to $|(I-\Sigma \Delta I)|$ and a criterion is applied based on energy and spin, as to whether the event will populate an isomeric level or the ground state.

Presently we are making the most simple of approximations, that all populations with spin greater than the average of isomer and ground state spins and with energies in excess of the isomer energies will populate the isomer, else the ground state will be populated (for cases where isomer spin exceeds ground state spin). Analogous algorithms are applied for cases where ground state spins exceed isomer spins. Some results of this very simplistic approach, inspired by early work of Vandenbosch and Huizenga [18,19], are shown in figure 10. This case, for protons incident on zirconium, shows good agreement between our model predictions and experimental data. To date we have also run our code for proton reactions on gold, and for some product isomers we see good agreement, though we also see poorer agreement in certain cases. As we proceed, our work will allow calculation of cases where more than one isomer may be formed, and we will explore more physical gamma ray cascade calculations for populating final states to replace the present guillotine cutoff - which however seems useful based on our early results of figure 10 . We presently include the option of using an yrast level for each "I" calculated using the rotating finite range model of Sierk. Emissions below the yrast level are forbidden; this gives a spin dependent level density within the framework of 
the Weisskopf-Ewing evaporation model, and also constrains somewhat the phase space available to the precompound decay chain.

\section{Conclusions}

The HMS model provides a powerful computational tool to simulate preequilibrium reactions. It is run in Monte Carlo mode, and so provides results on an event-by-event basis. This has the advantage of preserving full correlations in the reactions, so that one can determine exclusive as well as inclusive reaction cross sections. The Monte Carlo procedure also enables us to conserve and track momentum and energy in the multi-particle break-up process exactly. We showed how semiclassical arguments can be made that allow us to model spin transfer processes, which are particularly important in understanding isomer and residual nucleus gamma-ray cross sections. Finally, we also noted that use of the HMS approach allows us to avoid some of the questionable assumptions implicit within the exciton model, regarding the statistical population of particle-hole states.

Of course the main limitation of the present approach is its semiclassical nature. The spin tarnsfer effects can more rigorously computed using quantum approaches, such as the FKK model. Some early work in this direction was published in ref. [20]; more recent progress has been made by Kawano and others as they have undertaken research in modeling discrete gamma-rays measured using the GEANIE detector at LANSCE following preequilibrium reactions.

The authors would like to acknowledge useful discussions with Drs. Pavel Oblozinsky, Mike Herman, Phil Young, Toshihiko Kawano, Arjan Koning, Eric Bauge, and Patrick Talou. This work was supported by the United States Department of Energy Contract No. DE-AC52-06NA25396. Parts of this work were performed under a NNSA-CEA fundamental science collaboration agreement.

\section{References}

1. M. Blann, New precompound decay model, Phys. Rev. C 54, 1341 (1996).

2. M. Blann, M.B. Chadwick, New precompound model: Angular distributions, Phys. Rev. C 57, 233 (1998).
3. M. Blann, M.B. Chadwick, Precompound Monte Carlo model for cluster induced reactions, Phys. Rev. C 6203, 4604 (2000).

4. E. Gadioli, P.E. Hodgson, Pre-equilibrium nuclear reactions (Oxford University Press, Oxford, UK, 1992).

5. M. Blann, Annu. Rev. Nucl. Sci. 25, 123 (1975).

6. A. J. Koning, M.B. Chadwick, Microscopic two-component multistep direct theory for continuum nuclear reactions, Phys. Rev. C 56, 970 (1997).

7. M. Blann, H. Vonach, Phys. Rev. C 28, 1475 (1983).

8. T. Ericson, Adv. Phys. 9, 423 (1960).

9. J. Bisplinghoff, Phys. Rev. C 33, 1569 (1986).

10. M. Blann, Phys. Rev. Lett. 27, 337 (1971).

11. M. Blann, ref. [30] in Annu. Rev. Nucl. Sci. 25, 123 (1975).

12. R.W. West, Phys. Rev. 141, 1033 (1966).

13. A. Chevarier, N. Chevarier, A. Demeyer, G. Hollinger, R. Pertosa, Tran Minh Duc, Phys. Rev. C 8, 2155 (1973).

14. E. Gadioli, E. Gadioli Erba, J.J. Hogan, B.V. Jacak, Phys. Rev. C 29, 76 (1984).

15. P.G. Young, E.D. Arthur, M.B. Chadwick, Comprehensive nuclear model calculations: Theory and use of the GNASH code, in Proc. of the IAEA Workshop on Nuclear Reaction Data and Nuclear Reactors - Physics, Design, and Safety, Trieste, Italy, April 15 - May 17, 1996, edited by A. Gandini, G. Reffo (World Scientific Publishing, Ltd., Singapore, 1998), pp. 227404.

16. M. Chadwick, P. Young, R. MacFarlane, A. Koning, Evaluated nuclear data libraries up to $150 \mathrm{MeV}$ : Method for calculating recoils, in Proc. of Second International Conference on Accelerator-Driven Transmutation Technologies and Applications, Kalmar, Sweden, June 3-7, 1996, edited by H. Condé (Gotab, Stockholm, Sweden, 1997), pp. 483-489.

17. A. Koning, S. Hilaire, M. Duijvestijn, TALYS: Comprehensive nuclear reaction modeling, in Proc. of the Inter. Conf. on Nuclear Data for Science and Technology, Santa Fe, Sept. $26-$ Oct. 1, 2004, edited by R. Haight, M. Chadwick, T. Kawano, P. Talou (Amer. Inst. of Physics, New York, USA, 2005), pp. 1154-1159.

18. J.R. Huizenga, R. Vandenbosch, Interpretation of isomeric cross section ratios for $(n, \gamma)$ and $(\gamma, n)$ reactions, Phys. Rev. 120, 1305 (1960).

19. J.R. Huizenga, R. Vandenbosch, Phys. Rev. 120, 1313 (1960).

20. M.B. Chadwick, P.G. Young, P. Obložinský, A. Marcinkowski, Preequilibrium spin effects in Feshbach-Kerman-Koonin and exciton models and application to high-spin isomer production, Phys. Rev. C 49, R2885 (1994). 